\title{
Clinical Result of Low-Level Laser Therapy (LLLT) in Achilles Tendinitis
}

\author{
David $\mathrm{Ip}^{1}$ \\ ${ }^{1}$ MBBS FRCS(Edin)Orth FHKCOS FHKCOS(Rehab) FHKAM(Orth Surg), Asia Medical Pain Centre, $4^{\text {th }}$ Floor \\ Tower 2 Grand Plaza, Mong Kok Kowloon, Hong Kong \\ Correspondence: David Ip, MBBS FRCS(Edin)Orth FHKCOS FHKCOS(Rehab) FHKAM(Orth Surg), Asia \\ Medical Pain Centre, $4^{\text {th }}$ Floor Tower 2 Grand Plaza, Mong Kok Kowloon, Hong Kong. Tel: 852-2388-0389. \\ E-mail: ipdavid@hotmail.com.hk
}

\begin{tabular}{|c|c|}
\hline Received: December 29, 2015 & Online Published: February 23, 2016 \\
\hline doi:10.5539/apr.v8n2p40 & URL: http://dx.doi.org/10.5539/apr.v8n2p40 \\
\hline
\end{tabular}

\begin{abstract}
Objective: to study the clinical effects of low-level laser therapy (LLLT) in the clinical management of heel pain in sportsman having Achilles tendinitis

Materials and Methods: this is a prospective cohort study of 35 consecutive patients having Achilles tendinitis being referred to our pain centre after various sports, who did not respond to an initial minimum of 4 weeks of conventional physical therapy, and managed with low-level laser therapy for six consecutive weeks

Results: There was statistically significant $(\mathrm{p}<0.05)$ reduction of pain with respect to VAS scale, and pressure pain threshold, the post-treatment color Doppler revealed improved tendon vascularity, the clinical follow up was eight weeks

Conclusion: It is concluded that LLLT has clinical efficacy in the short-term treatment of Achilles tendinitis in sportsman
\end{abstract}

Keywords: Achilles tendinitis, heel pain, laser therapy, outcome

\section{Introduction}

The importance of low level laser dates back to the sixties with the important works of Endre Mester. The various clinical applications in recent years of Low Level Laser Therapy (LLLT) were mainly based on additional scientific works concerning the effect of low-level lasers which exert a positive influence on cellular-molecular level on the fibroblast (Van Breugel \& Bär, 1992) and collagen synthesis (Lam et al., 1986).

As far as Achilles tendinitis is concerned, most clinicians use non-steroidal anti-inflammatory medications, local steroid injections, and bedside ultrasound as treatment. The former two treatment modalities represent symptomatic treatment without bio-modulation effects as do low-level lasers; while ultrasound treatment afford neither anti-inflammatory nor bio-modulation effects on a cellular-molecular level.

Although there have been clinical studies of the use of LLLT in various forms of arthritis (Brosseau et al., 2000), there is a relative paucity of clinical studies of LLLT on tendinitis. The objective of the current study is to investigate the efficacy of LLLT therapy in the management of Achilles tendinitis in young sportsman

\section{Materials and Methods}

The study population consisted of a series of consecutive unselected thirty five patients with forty painful Archilles tendons diagnosed clinically and confirmed by ultrasound examination of the affected tendon group. This represents a group of patients who failed to respond to not less than 4 weeks of conventional physical therapy before being referred to our tertiary referral pain centre. The mean age of the patient population was 27 (range 23 to 32).

Each subject was clinically examined by the same Orthopaedic surgeon to minimize the inter-observer error as to the spot of maximum tenderness of the tendon. The Visual Analogue Scale (VAS) of Pain was used to score the level of pain during the first clinic visit as well as on subsequent visits to monitor the response to treatment. Ultrasonography (Siemens Sonoline G60) was performed to ensure the exact location of tendon pathology prior to initiation of therapy, and was performed by the same Orthopaedic surgeon who performed the clinical examination 
and recording the VAS Pain Score. Tendon vascularisation was examined by power Doppler.; peritendinous or intratendinous arterial blood flow velocity was also measured. Informed consent was obtained prior to commencement of therapy.

LLLT of $810 \mathrm{~nm}$ wavelength emitting from from GaAIAs semi-conductor laser device with $5.4 \mathrm{~J}$ per point, power density $20 \mathrm{~mW} / \mathrm{cm} 2$ was employed. The treatment regime consisted of three sessions of treatment per week for six consecutive weeks. Each treatment session lasted 180 seconds. Upon completion of therapy, each patient had weekly follow up to monitor the response to treatment and to document any side effects for eight consecutive weeks. Since it is not uncommon for patients with Achilles tendinitis to present with symptom of one lower limb rather than both lower limbs, it is technically difficult to arrange for a placebo group.

\section{Results}

All subjects in the study population completed the treatment regimen with good compliance, there was no defaults. There were twenty six right side and fourteen left side Achilles tendons being treated in this study. Five patients had bilateral tendon involvement.

There was statistically significant reduction of VAS pain score for the heel pain compared to the VAS pain score during the initial clinical examination $(p<0.05)$. The pressure pain threshold as measured by digital pressure algometry also showed improvement after active treatment by LLLT $(p<0.05)$; the above results suggested the positive clinical responses of LLLT managed painful tendons were unlikely to have arisen by chance

In addition, the peritendinous and intra-tendinous blood flow as measured by colour Doppler were improved after active LLLT administration upon completion of therapy at the six week mark as compared to before initiation of therapy, providing further evidence of the objective effect of the laser therapy. The follow-up period of this study after low level laser treatment was completed was eight weeks and the positive clinical response was maintained for this period with no clinical relapse nor deterioration. No side effects was observed throughout the study.

\section{Discussion}

Over the past years, there have been many placebo-controlled studies being published on the effects of LLLT. These articles also showed the favorable anti-inflammatory effect of LLLT (Bjordal, Johnson, Iversen, Aimbire, \& Lopes-Martins, 2006; Bjordal, Lopes-Martins, \& Iversen, 2006). However, as the current author pointed out in published medical texts, there are more abundant medical literature on the treatment of painful joints rather than tendons (Ip, 2007).

Traditional methods of treatment of painful Achilles tendonitis which involves the heel tendo-archilles include non-steroidal anti-inflammatory agents, steroid injections, ultrasound therapy, and electrical stimulation therapy. None of the above therapy harbor the ability of bio-modulation effect offered by LLLT, such as improvement in microcirculation, as well as an up-regulation of several genes involved in energy metabolism and oxidative phosphorylation thus stimulating an increase in ATP production, which in turn regulates other cellular processes leading to normalization of biological functions at the cellular level (Hashmi et al., 2010). This is in sharp contrast to the biophysical effects of therapeutic ultrasound devices that do not even have anti-inflammatory effects let alone biomodulation effects (Baker, Robertson, \& Duck, 2001).

In conclusion, based on the results of the current prospective cohort study, LLLT can be considered as a viable non-invasive treatment option in painful heel pain in sportsman caused by Achilles tendinitis. Future larger scale trials are needed however to determine optimal dose ranges and intervals between applications.

\section{Acknowledgement}

No grants or supports was received from any company nor institution in connection with the present study

\section{References}

Baker, K. G., Robertson, V. J., \& Duck, F. A. (2001). A review of therapeutic ultrasound: biophysical effects. Phys Ther, 811351-1358.1358

Bjordal, J. M., Johnson, M. I., Iversen, V., Aimbire, F., \& Lopes-Martins, R. A. (2006) Photoradiation in acute pain: a systematic review of possible mechanisms of action and clinical effects in randomized placebo-controlled trials. Photomed. Laser Surg, 24, 158-68.

Bjordal, J. M., Lopes-Martins, R. A., \& Iversen, V. V. (2006). A randomised, placebo controlled trial of low level laser therapy for activated Achilles tendinitis with microdialysis measurement of peritendinous prostaglandin E2 concentrations. Br. J. Sports Med, 40, 76-80. 
Brosseau, L., Welch, V., Wells, G., Tugwell, P., de Bie, R., \& Gam, A. (2000) Low level laser therapy for osteoarthritis and rheumatoid arthritis: a meta-analysis. J Rheumatol Aug, 27(8), 1961-9.

Hashmi, J. T., Huang, Y. Y., Osmani, B. Z., Sharma, S. K., Naesar, M. A., \& Hamblin, M. R. (2010). Role of low-level laser therapy in Neuro-rehabilitation. American Academy of Physical Medicine and Rehabilitation Dec 2(12 Suppl 2), S292-305.

Ip, D. (2007). Orthopedic Rehabilitation, Assessment and Enablement. Springer-Verlag Heidelberg Germany Chapter 2.

Lam, T. S., Abergel, R. P., Meeker, C. A., Castel, J. C., Dwyer, R. M., \& Uitto, J. (1986). Laser stimulation of collagen synthesis in human skin fibroblasts cultures. Lasers Life Sci, 1, 61-77.

Van Breugel, H. H. F. I., \& Bär, P. R. D. (1992). Power density and exposure time of He-Ne laser irradiation are more important than total energy dose in photo-biomodulation of human fibroblasts in vitro. Lasers Surg. Med, 12, 528-537.

\section{Copyrights}

Copyright for this article is retained by the author(s), with first publication rights granted to the journal.

This is an open-access article distributed under the terms and conditions of the Creative Commons Attribution license (http://creativecommons.org/licenses/by/3.0/). 